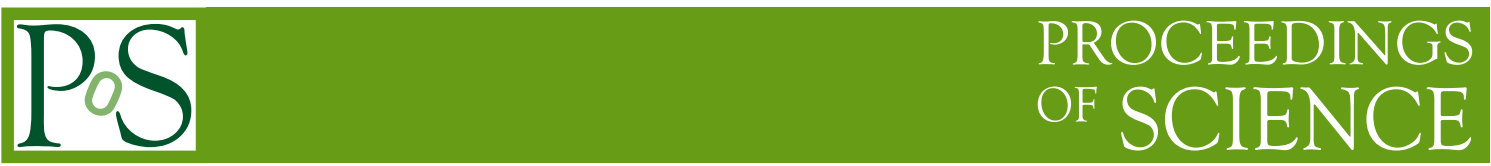

\title{
Bottomonium results and prospects at Belle II
}

\author{
Gian Luca Pinna Angioni ${ }^{a, *}$ on behalf of the Belle II Collaboration \\ ${ }^{a}$ Universita di Torino - INFN, Sezione di Torino. Via P. Giuria 1, 10125 Torino, Italy \\ E-mail: gianluca.pinnaangioni@to.infn.it
}

The Belle II experiment at the SuperKEKB energy-asymmetric $e^{+} e^{-}$collider is an upgrade of the $B$ factory facility at KEK in Tsukuba, Japan. The experiment began operation in 2019 and aims to record 50 times more data than its predecessor. Belle II is uniquely capable of studying the so-called "XYZ" particles: heavy exotic hadrons consisting of more than three quarks. First discovered by Belle, these now number in the dozens, and represent the emergence of a new category within quantum chromodynamics. We present recent results in new Belle II data, and the future prospects to explore both exotic and conventional bottomonium physics.

\footnotetext{
*** The European Physical Society Conference on High Energy Physics (EPS-HEP2021), *** *** 26-30 July $2021 * * *$

*** Online conference, jointly organized by Universität Hamburg and the research center DESY ***
}

\footnotetext{
${ }^{*}$ Speaker
} 


\section{Introduction}

The Belle II experiment [1] is installed at the SuperKEKB collider [2] an asymmetric $e^{+} e^{-} B$ meson factory, and even if its primary goal is to study CP-violation and rare decays, it has great potential in other topics such bottomonia. The Belle II detector is a major upgrade of the previous Belle experiment and consists of several sub-detectors that were either upgraded or completely replaced. The aim of the upgrade was to obtain similar performance as Belle at luminosities up to 40 times higher.

In $e^{+} e^{-}$colliders, bottomonium (along with bottomonium-like exotic states) can be produced in different ways. $\mathrm{J}^{P C}=1^{--}$states can be produced directly if the mass of the states correspond to the center-of-mass energy $(\sqrt{s})$, or, via intial-state-radiation (ISR) process if the mass is lower. From the $\mathrm{J}^{P C}=1^{--}$states it is then possible to access other states through both radiative transitions and hadronic transitions such $\pi \pi, \eta$ and $\omega$.

Quarkonium spectroscopy has been investigated in detail by the first generation of $B$-factories and other experiments and it turns out to be rich in new production mechanisms, new transitions and unexpected states of an exotic nature. The discovery of the $X(3872)$ [3] opened the way to a plethora of exotic quarkonium-like particles in the charmonium sector and the following discoveries of the $Z_{b}(10610)$ and $Z_{b}(10650)$ states $[4,5]$ hints that a similar family of particles could exist also in the bottomonium one. Within the predicted bottomonium spectrum there are missing states below $B \bar{B}$ threshold, e.g., spin-singlet member of the $1 \mathrm{D}$ multiplet, all members of the $2 \mathrm{D}$ and $1 \mathrm{~F}$ multiplets, that still need to be identified. Moreover the bottomonium sector offers an interesting doorway of the physics beyond the Standard Model such as lepton flavor violation, lepton flavor universality, dark matter and low mass Higgs bosons.

\section{Bottomonium at $\Upsilon(4 S)$}

Belle II started taking data in spring 2018 and recorded to date an integrated luminosity of 213 $\mathrm{fb}^{-1}$ at the energy of the $\Upsilon(4 S)$. The goal is to collect 50 times more data $\left(50 \mathrm{ab}^{-1}\right)$ than Belle. Data collected at the $\Upsilon(4 S)$ energy are only a small part of the bottomonium physics program. The branching fraction of $\Upsilon(4 S)$ to $B \bar{B}$ is nearly $100 \%$, leaving little opportunity for decays to other bottomonium states or decays useful for new physics studies. One way to overcome this obstacle is to exploit ISR to reach lower states such $\Upsilon(2 S)$ or $\Upsilon(3 S)$ albeit with a cross section on the order of $10 \mathrm{pb}$ while the $B \bar{B}$ production is $\sim 1.2 \mathrm{nb}$.

A recent study assessed the sensitivity on the lepton universality measurement in the channel $\Upsilon(1 S) \rightarrow \ell \ell$ with $\ell=\tau, e$ via ISR at the energy of $\Upsilon(4 S)$. The work estimated the statistical uncertainty as a function of the integrated luminosity and compared the result with a measurement from BaBar performed at the $\Upsilon(3 S)$ [6]. The study is performed with a Monte Carlo (MC) simulation and covers an integrated luminosity range up to the design value of $50 \mathrm{ab}^{-1}$. In Fig. 1 the statistical uncertainty of $e^{+} e^{-} \rightarrow \gamma_{\mathrm{ISR}}(\Upsilon(1 S) \rightarrow \tau \tau)$ is shown together with the value of $0.8 \%$ from the BaBar analysis. The statistical uncertainty obtained with $50 \mathrm{ab}^{-1}$ is $2.5 \%$ which is not sufficient for a competitive analysis. This result shows how it's important to take data below the $\Upsilon(4 S)$ to perform these type of studies. 


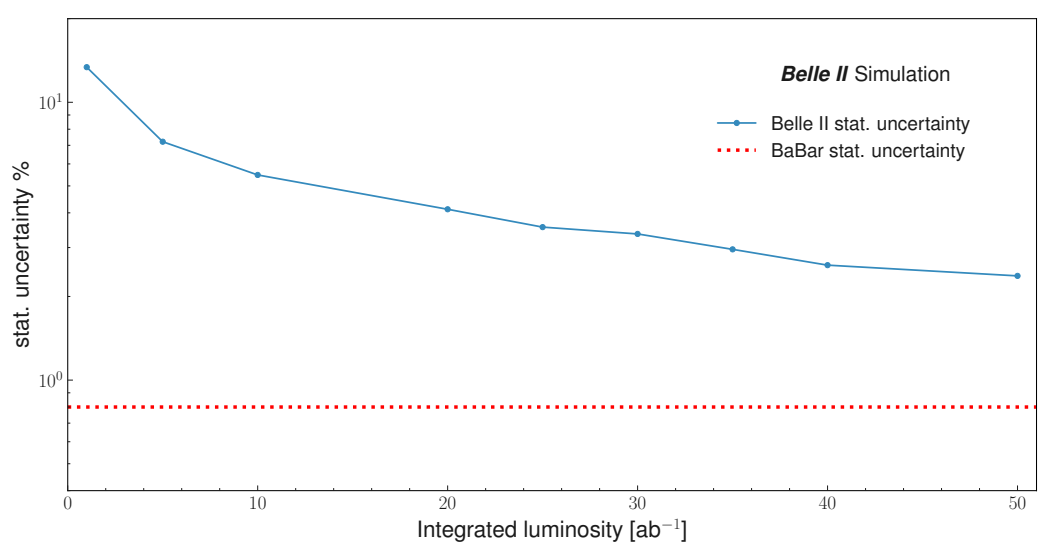

Figure 1: Projection of statistical uncertainty on the cross section of $e^{+} e^{-} \rightarrow \gamma_{\mathrm{ISR}}(\Upsilon(1 S) \rightarrow \tau \tau)$ as a function of the integrated luminosity. The dashed red line shows the statistical uncertainty of the competitive analysis from BaBar [6].

The currently available integrated luminosity of $213 \mathrm{fb}^{-1}$ is insufficient for competitive results, but nevertheless early bottomonium analyses are performed. These studies are crucial to assert the capability of the new detector to perform as well as Belle or better. Moreover many of these analyses are also essential in preparation for the data taking at different energies as they can be transferred with minimal changes.

One example is the search for $\Upsilon$ dipion transitions that has been recently carried out with a data set of $72 \mathrm{fb}^{-1}$. The analysis reconstructs $\Upsilon(m S) \rightarrow \pi^{+} \pi^{-}\left[\Upsilon(p S) \rightarrow \mu^{+} \mu^{-}\right]$decays, where $m=$ 2,3,4 and $p=1,2$ with $m>p$. The direct transitions $\Upsilon(4 S) \rightarrow \pi^{+} \pi^{-} \Upsilon(1 S, 2 S)$ have been observed and thanks to the ISR process also $\gamma_{\mathrm{ISR}} \Upsilon(3 S) \rightarrow \pi^{+} \pi^{-} \Upsilon(1 S, 2 S)$ and $\gamma_{\mathrm{ISR}} \Upsilon(2 S) \rightarrow \pi^{+} \pi^{-} \Upsilon(1 S)$ processes have been detected. In Fig. 2 are shown the distributions of the main variables used in the analysis where the different signal transitions are clearly visible. It's useful to make the comparison with the Belle results where, with a data set of $496 \mathrm{fb}^{-1}$, the $\gamma_{\mathrm{ISR}} \Upsilon(3 S) \rightarrow \pi^{+} \pi^{-} \Upsilon(2 S)$ was not visible [7]. This can be explained by the retention of lower-momentum pion candidates in Belle II compared to Belle. This early study, made with only a small data set, already shows the good performance of Belle II and the potential of this channel.

Another MC study has been performed to asses the performance of Belle II by reproducing the Belle discovery of the $h_{b}(1 P)$ in the channel $\Upsilon(4 S) \rightarrow \eta(\rightarrow \gamma \gamma) h_{b}(1 P)$ [11]. The study is performed by reconstructing the $\eta$ in the $\gamma \gamma$ decay channel and looking at its recoil mass. Since no exclusive decays of the $h_{b}(1 P)$ into stable particles are known, the $h_{b}(1 P)$ is not reconstructed. Fig. 3 shows the result of a fit example on the $\gamma \gamma$ recoil mass and the expected statistical significance of the $\Upsilon(4 S) \rightarrow \eta h_{b}(1 P)$ ) signal as function of the integrated luminosity. The Belle result is also shown. The better result by Belle II is due to an improved analysis, and shows that despite the increased background, Belle II can outperform Belle by means of more refined selections. 


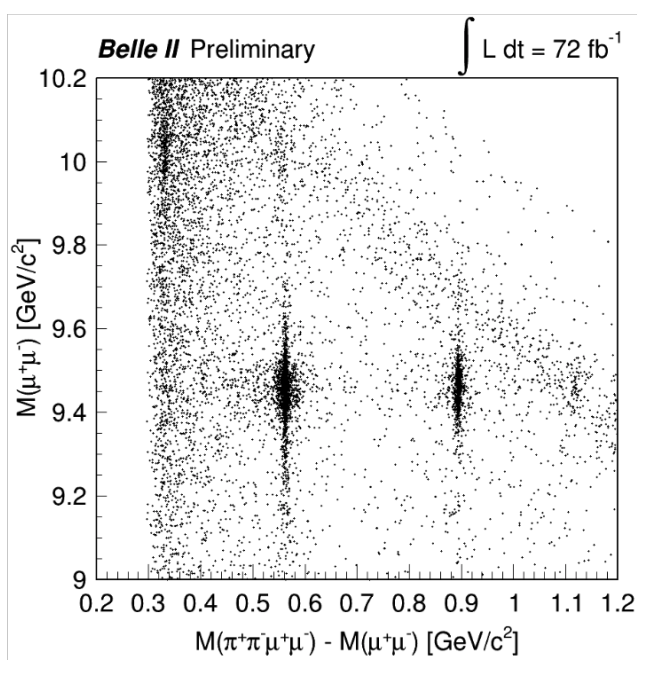

(a)

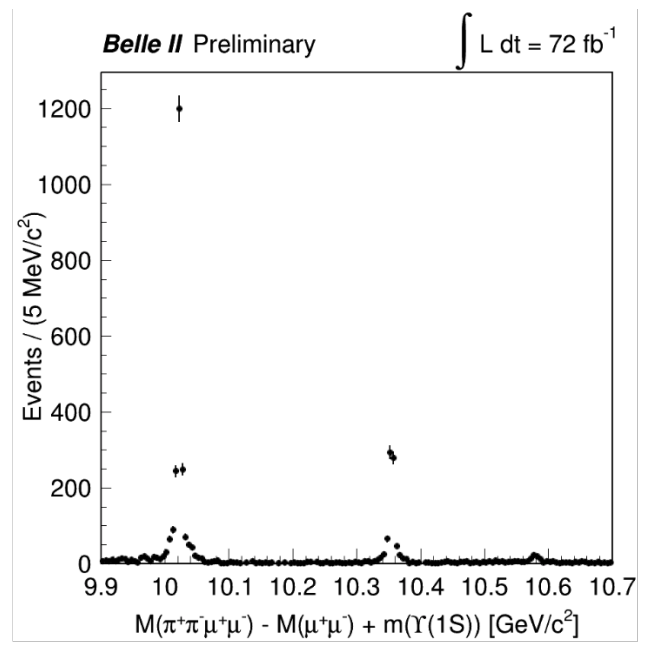

(b)

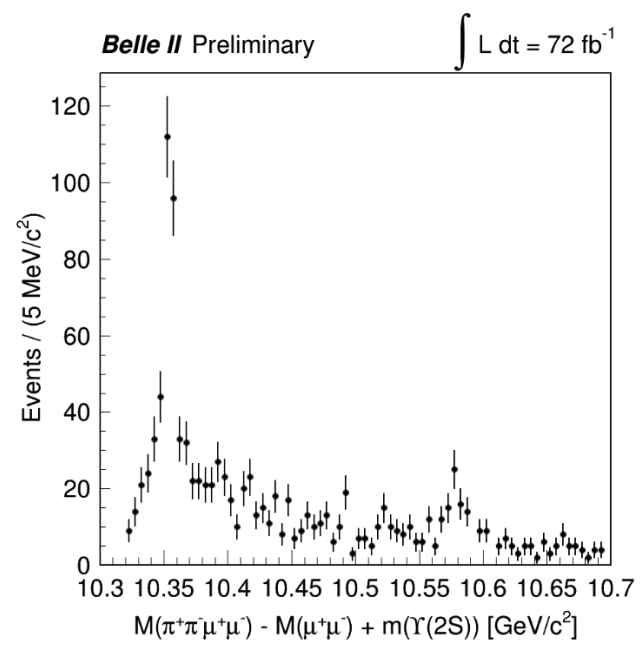

(c)

Figure 2: a) Distributon of $M(\mu \mu)$ versus $M\left(\pi^{+} \pi^{-} \mu^{+} \mu^{-}\right)-M\left(\mu^{+} \mu^{-}\right)$. The clusters of points represent signal transitions. b) Distribution of $M\left(\pi^{+} \pi^{-} \mu^{+} \mu^{-}\right)-M\left(\mu^{+} \mu^{-}\right)+m(\Upsilon(1 S))$ with a requirement of $\left|M\left(\mu^{+} \mu^{-}\right)-m(\Upsilon(1 S))\right|<75 \mathrm{MeV} / \mathrm{c}^{2}$, where $m(\Upsilon(1 S))$ represents the nominal $\Upsilon(1 S)$ mass. c) Distribution of $M\left(\pi^{+} \pi^{-} \mu^{+} \mu^{-}\right)-M\left(\mu^{+} \mu^{-}\right)+m(\Upsilon(2 S))$ with a requirement of $\left|M\left(\mu^{+} \mu^{-}\right)-m(\Upsilon(2 S))\right|<75 \mathrm{MeV} / \mathrm{c}^{2}$, where $m(\Upsilon(2 S))$ represents the nominal $\Upsilon(2 S)$ mass.

\section{Belle II potential}

Across all experiments, the currently available data sets relevant for bottomonium physics at center-of-mass energies different from the $\Upsilon(4 S)$ resonance are $O(10) \mathrm{fb}^{-1}$ for each of the $\Upsilon(1 S, 2 S, 3 S, 6 S)$ resonances, about $121 \mathrm{fb}^{-1}$ at the $\Upsilon(5 S)$ resonance, and less than $1 \mathrm{fb}^{-1}$ at intermediate energies. Given the small size of the existing data sets and the current luminosity at SuperKEKB, only a relatively small amount of collision time would be needed to collect non- $\Upsilon(4 S)$ samples an order of magnitude larger. Belle II and SuperKEKB have an exceptional opportunity both 


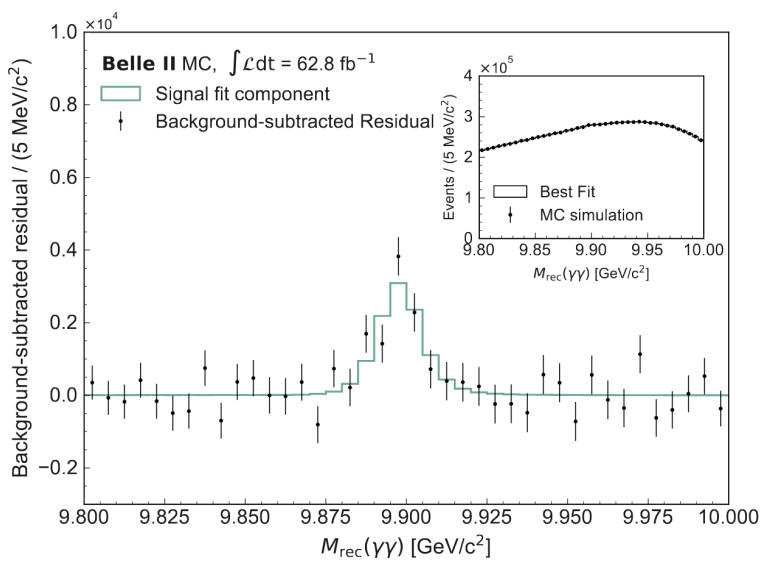

(a)

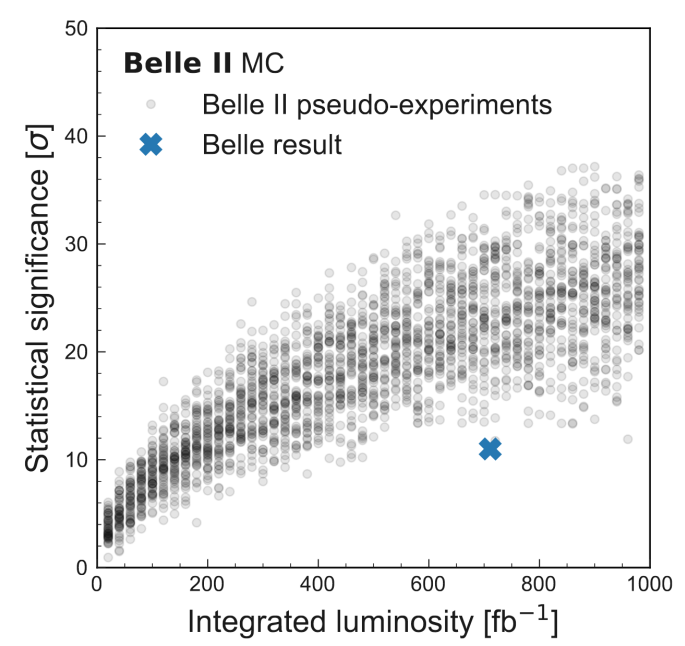

(b)

Figure 3: a) Result of the fit of the rescaled MC when the expected number of $\Upsilon(4 S) \rightarrow h_{b}(1 P), 12000$, is included. The inset shows the fitted distribution, while the main plot shows the residual after subtracting the background. b) Expected significance as function of the luminosity collected. Each point is the result of a the fit of a pseudo-experiment. The blue cross indicates the Belle result.

above and below the $\Upsilon(4 S)$ energy and a dedicated bottomonium program is under construction.

\subsection{The $\Upsilon(10753)$ case}

Recently, the Belle Collaboration reanalyzed the cross section of $e^{+} e^{-} \rightarrow \Upsilon(n S) \pi^{+} \pi^{-}(n=$ $1,2,3)$ and updated their measurements to supersede the previous result $[8,9]$. In the analysis a new structure near $10.75 \mathrm{GeV}$ has been found in the $\Upsilon(n S) \pi^{+} \pi^{-}(n=1,2,3)$ invariant mass spectrum. This new state, called $\Upsilon(10753)$ in PDG [10], can't be easily explained as a conventional bottomonium state as all the calculations of the mass spectrum of bottomonium family don't fit with its mass. Many different explanations of the $\Upsilon(10753)$ were given, both conventional [12-15] and exotic [16-19].

Given the importance to investigate this new state and the energy region around it, a dedicated 
early data taking program has been prepared for the end of 2021. Approximately $10 \mathrm{fb}^{-1}$ of data are planned on the resonance and three scan points for $B \bar{B}$ decomposition studies $\left(1.5 \mathrm{fb}^{-1}\right.$ at 10.657 $\mathrm{GeV}, 3.5 \mathrm{fb}^{-1}$ at $10.706 \mathrm{GeV}$ and $2 \mathrm{fb}^{-1} 10.810 \mathrm{GeV}$ ).

The measurement and the MC study shown in these proceedings, $\Upsilon(n S)$ dipion transitions and the $\Upsilon(n S) \rightarrow \eta h_{b}(1 P)$ decay respectively, are both easly transferable to the $\Upsilon(10753)$ energy and they are key to the study of the new state.

\section{Summary}

The Belle II experiment has recently begun data collection and it's proving to perform as expected. Together with the capability of SuperKEKB, Belle II has a unique opportunity to study quarkonium(-like) particles and make an impact in this sector in the years to come.

\section{References}

[1] T. Abe et al. [Belle-II], Belle II Technical Design Report

[2] K. Akai et al. [SuperKEKB], SuperKEKB Collider, Nucl. Instrum. Meth. A 907 (2018), 188-199

[3] S. K. Choi et al. [Belle], Observation of a narrow charmonium-like state in exclusive $B^{ \pm} \rightarrow$ $K^{ \pm} \pi^{+} \pi^{-} J / \psi$ decays, Phys. Rev. Lett. 91 (2003), 262001

[4] C. Oswald and T. K. Pedlar, Results in $B_{s}$ physics and bottomonium spectroscopy using the Belle $\Upsilon$ (5S) data, Mod. Phys. Lett. A 28 (2013), 1330036

[5] P. Krokovny et al. [Belle], First observation of the $Z_{b}^{0}(10610)$ in a Dalitz analysis of $\Upsilon(10860)$ $\rightarrow \Upsilon(n S) \pi^{0} \pi^{0}$, Phys. Rev. D 88 (2013) no.5, 052016

[6] J. P. Lees et al. [BaBar], Precision measurement of the $\mathcal{B}\left(\Upsilon(3 S) \rightarrow \tau^{+} \tau^{-}\right) / \mathcal{B}\left(\Upsilon(3 S) \rightarrow \mu^{+} \mu^{-}\right)$ ratio, Phys. Rev. Lett. 125 (2020), 241801

[7] E. Guido et al. [Belle], Study of $\eta$ and dipion transitions in $\Upsilon(4 S)$ decays to lower bottomonia, Phys. Rev. D 96 (2017) no.5, 052005

[8] R. Mizuk et al. [Belle], Observation of a new structure near $10.75 \mathrm{GeV}$ in the energy dependence of the $e^{+} e^{-} \rightarrow \Upsilon(n S) \pi^{+} \pi^{-}(n=1,2,3)$ cross sections, JHEP 10 (2019), 220

[9] D. Santel et al. [Belle], Measurements of the $\Upsilon(10860)$ and $\Upsilon(11020)$ resonances via $\sigma\left(e^{+} e^{-} \rightarrow \Upsilon(n S) \pi^{+} \pi^{-}\right)$, Phys. Rev. D 93 (2016) no.1, 011101

[10] P.A. Zyla et al. [Particle Data Group], Review of Particle Physics, PTEP 2020 (2020) no.8, $083 \mathrm{C} 01$

[11] U. Tamponi et al. [Belle], First observation of the hadronic transition $\Upsilon(4 S) \rightarrow \eta h_{b}(1 P)$ and new measurement of the $h_{b}(1 P)$ and $\eta_{b}(1 S)$ parameters, Phys. Rev. Lett. 115 (2015) no.14, 142001 
[12] Q. Li, M. S. Liu, Q. F. Lü, L. C. Gui and X. H. Zhong, Canonical interpretation of $\Upsilon$ (10750) and $\Upsilon(10860)$ in the $\Upsilon$ family, Eur. Phys. J. C 80 (2020) no.1, 59

[13] W. H. Liang, N. Ikeno and E. Oset, $\Upsilon(n l)$ decay into $B^{(*)} \bar{B}^{(*)}$, Phys. Lett. B 803 (2020), 135340

[14] B. Chen, A. Zhang and J. He, Bottomonium spectrum in the relativistic flux tube model, Phys. Rev. D 101 (2020) no.1, 014020

[15] Y. S. Li, Z. Y. Bai, Q. Huang and X. Liu, Hidden-bottom hadronic decays of $\Upsilon$ (10753) with a $\eta($ ') or w emission, Phys. Rev. D 104 (2021) no.3, 034036

[16] Z. G. Wang, Vector hidden-bottom tetraquark candidate: Y(10750), Chin. Phys. C 43 (2019) no. 12,123102

[17] P. Bicudo, N. Cardoso, L. Müller and M. Wagner, Computation of the quarkonium and mesonmeson composition of the $\Upsilon(n S)$ states and of the new $\Upsilon(10753)$ Belle resonance from lattice QCD static potentials, Phys. Rev. D 103 (2021) no.7, 074507

[18] A. Ali, L. Maiani, A. Y. Parkhomenko and W. Wang, Interpretation of $Y_{b}(10753)$ as $a$ tetraquark and its production mechanism, Phys. Lett. B 802 (2020), 135217

[19] J. F. Giron and R. F. Lebed, Spectrum of the hidden-bottom and the hidden-charm-strange exotics in the dynamical diquark model, Phys. Rev. D 102 (2020) no.1, 014036 